\title{
Experimental Technique for Dynamic Fragmentation of Liquid- Driving Expanding Ring
}

\author{
Jia Zhang, Yuxuan Zheng ${ }^{*}$, Fenghua Zhou, and Jun Liu \\ MOE Key Laboratory of Impact and Safety Engineering, Ningbo University, Ningbo, Zhejiang, China
}

\begin{abstract}
Expanding ring experiment is an important method for dynamic fragmentation of solid under 1D tensile loading. Based on the split Hokinson pressure bar (SHPB), a liquid-driving experimental technology was developed for conducting expanding ring tests. The loading fixture includes a hydraulic cylinder filled with water, which is pushed by a piston connected to the input bar. As the water is driven, it expands the metallic ring specimen in the radial direction. The approximately incompressible property of the water makes it possible to drive the specimen in very high radial velocity by low velocity movement of piston, according to the large sectional area ratio of the cylinder to specimen. Using liquid-driving expanding ring device, 1060 aluminum rings (ductile materials)/PMMA rings (brittle materials) were fragmented and the fragments were recovered. Impact deformation of free-flying fragments was avoided through the use of "sample soft-capture" technology. The fragmentation process was observable by high speed camera through modifying the driving direction of the water. From the observations of the fracture morphology and the residual internal cracks of the recovered fragments, it is concluded that the fracture of the rings is caused by the circumferential tensile stress.
\end{abstract}

\section{Introduction}

The phenomena of dynamic fragmentation frequently occur during hypervelocity impacts. Understanding the fragmentation properties of solids is important to researchers in the fields of physics, mechanics, aerospace and defence engineering. The expanding ring technique is an effective way of determining the process of the fragmentation. To achieve the dynamic fragmentation of solids, the stress state in the specimen is uniaxial stress, high strain rate and uniform tension as much as possible. Because the specimen is under onedimensional tensile loading and there is no boundary along the circumferential direction in the process of rapid expansion. The expanding ring experiments include electromagnetic loading and explosive loading. Explosives are applied to drive the expanding ring, which was pioneered by Johnson and his partners [1] in 1963. With this method, Hoggatt and Recht [2] obtained the dynamic uniaxial tensile stress-strain data for several ductile materials. Experimental investigation of tensile fracture and fragmentation was firstly reported by Niordson [3], who designed an electromagnetic loading scheme to drive the expanding ring arrangement. The capability of the expanding ring method was fully exploited by Grady and Benson [4] who performed numerous experiments on OFHC copper and 1100-O aluminum. Gourdin [5] extended the applicability of the expanding ring experiment by employing conducting materials as pushers for testing of non-conducting specimens. With the electromagnetic loading technique,
Zhang and Ravi-Chandar [6, 7] reported the mechanics and physics of necking and fragmentation of ductile materials in a series of studies.

In previous studies, we [8] reported a new experimental approach based on the SHPB for the studies on the process of the dynamic fragmentation. The loading fixture includes a hydraulic cylinder full of incompressible fluid, which is pushed by a piston connected to the input bar. As the liquid is driven, it compresses and expands the metallic ring specimen in the radial direction. The approximately incompressible property of the liquid makes it possible to transfer a low piston-velocity to a very high radial velocity of the specimen, as the sectional areas of the cylinder narrows extremely. Using this experimental technology the ring specimens made of aluminum alloy were dynamically expanded and fragmentized. However, The expansion process of the specimen cannot be observed due to the specimen sealed by the device.

In this paper, impact deformation of free-flying fragments was avoided through the use of "sample softcapture" technology. The fragmentation process is observable by high speed camera through modifying the driving direction of the fluid. The specimen surface expansion rate was measured using laser interference device VISAR. Using the improved liquid-driving expanding ring device, 1060 aluminum rings (ductile materials) and PMMA rings (brittle materials) were fragmented and the fragments were recovered. 


\section{Expanding ring experiment}

\subsection{Experimental method}

A schematic diagram of such an apparatus is shown in Fig.1. The impact loading fixture, as a large specimen, is installed between the concentric input bar and output bar of the SHPB of diameter $74 \mathrm{~mm}$. The driven fluid is water. Literature [9] gives a detailed description of the experimental device. The specimen is in the form of a thin circular ring of inner diameter $\mathrm{d}=32 \mathrm{~mm}$, Outer diameter $\mathrm{D}=35 \mathrm{~mm}$ and cross sectional dimensions 1.5 $\mathrm{mm}$ and $1.5 \mathrm{~mm}$ shown in Fig. 2.

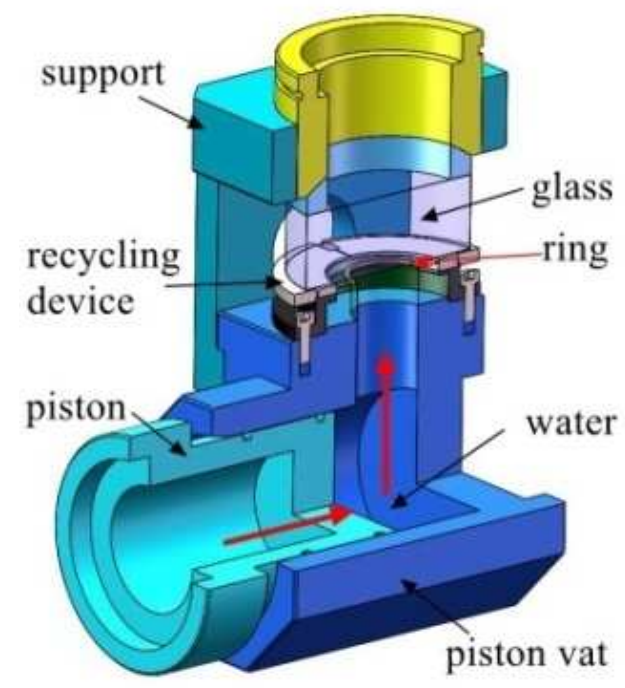

Fig. 1. Liquid-driving expanding ring experimental schematic diagram.

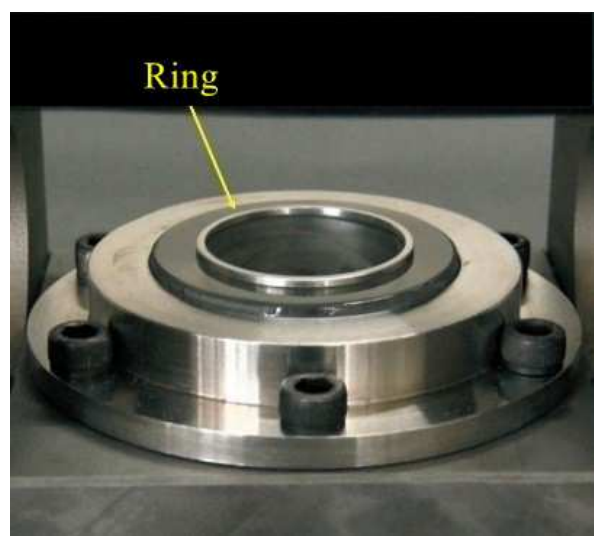

Fig. 2. Liquid-driving expanding ring.

The bullet of the Hopkinson press system hits the incident bar and the bar impacts the piston. The water was used as the transmission medium. The double Oring sealed on the piston can effectively prevent the leakage of liquid in the high-speed impact process. The ring specimen is sealed in the liquid cylinder and expands outwards under the impact of high pressure water. The transparent quartz glass is fixed on the top of the expanding ring. The fragmentation process was observable by high speed camera through modifying the driving direction of the water.

\subsection{Dynamic tensile fragment process}

\subsubsection{0 aluminum}

Fig. 3 shows the deformation and fracture (fragmentation) process of the 1060 aluminum ring under impact loading. Under the axial tension loading, we divide the fragmentation process into several parts: elastic deformation, uniformity plastic deformation, unstable plastic deformation (necking), onset of the damage, fragmentation and free flight. Expatiation: before time $30 \mu \mathrm{s}$, the bar is under uniform strain-rate, the plastic deformation is the same at any point; unstable plastic flow occurs at time $70 \mu \mathrm{s}$, and multiple necks appear; the necking grows with time, and at time $100 \mu \mathrm{s}$, the bar fragment into 6 pieces.
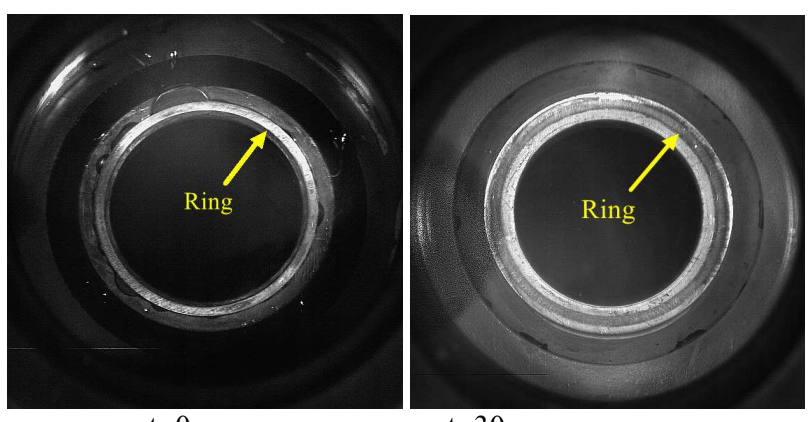

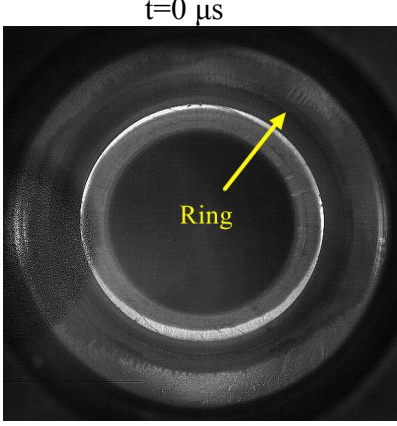

$\mathrm{t}=70 \mu \mathrm{s}$

\section{$\mathrm{t}=30 \mu \mathrm{s}$}

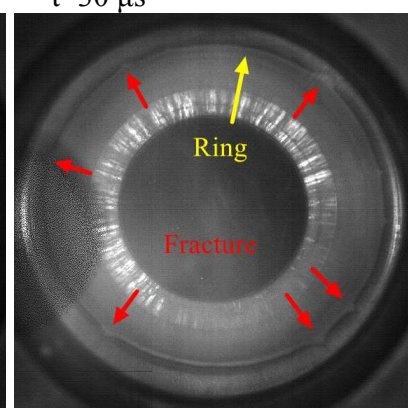

$\mathrm{t}=100 \mu \mathrm{s}$
Fig. 3. Typical tension and fragmentation process of the ductile expanding ring.

In the experiment, the radial expansion velocity history of the specimen is recorded by VISAR laser interferometer shown in Fig. 4. The expansion velocity divides the fragmentation process into three parts: boost phase, decelerating phase, constant speed. The start point of the time under the constant speed stage is the fracture time of the expanding ring. 


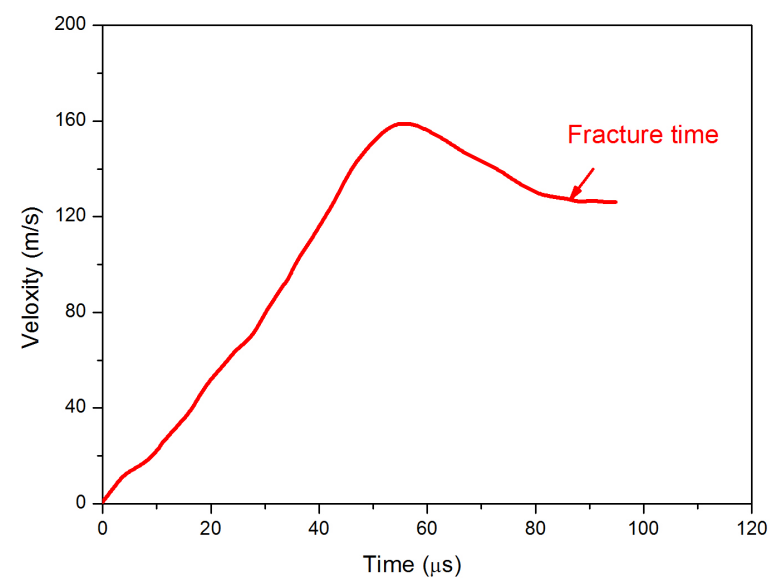

Fig. 4. Typical radial velocity history curves of 1060 aluminum ring.

\subsubsection{PMMA}

Fig. 5 shows the deformation and fracture (fragmentation) process of the PMMA ring under impact loading. Under the axial tension loading, we divide the fragmentation process into several parts: elastic deformation, onset of the damage, crack development, fragmentation and free flight. Expatiation: at time $38 \mu \mathrm{s}$, the crack began to appear, at time $42 \mu \mathrm{s}$, multiple crack developed; at time $58 \mu \mathrm{s}$, the ring fragment into lots of pieces.
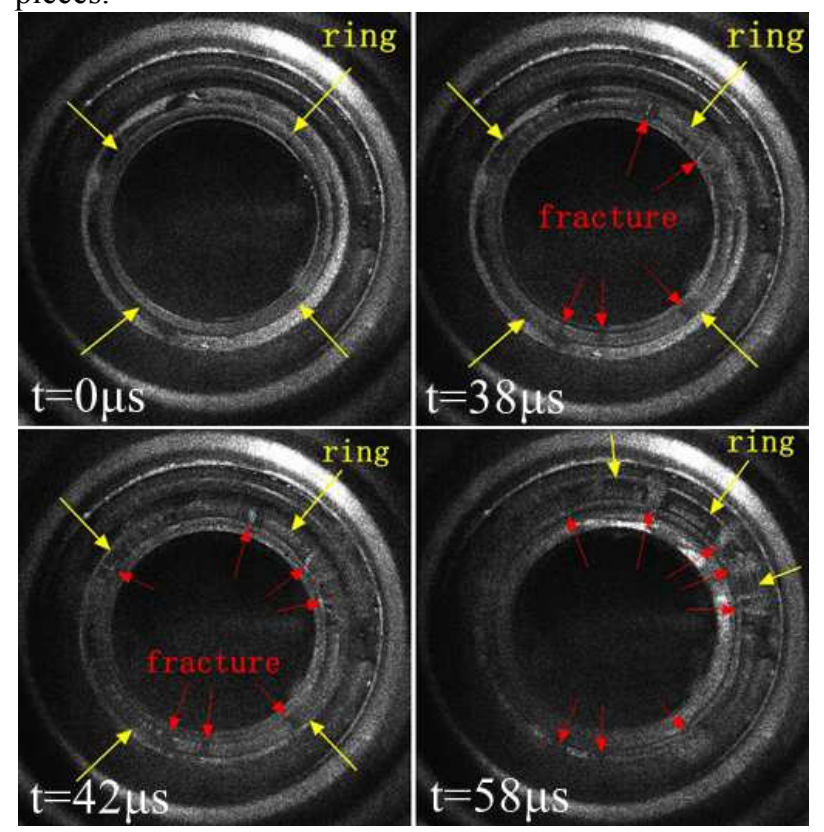

Fig. 5. Typical tension and fragmentation process of the brittle expanding ring.

In the experiment, the radial expansion velocity history of the specimen is recorded by VISAR laser interferometer shown in Fig. 6. The expansion velocity divides the fragmentation process into two parts: boost phase, vibration stage. The fracture time of the expanding ring is in the boost phase and not obvious.

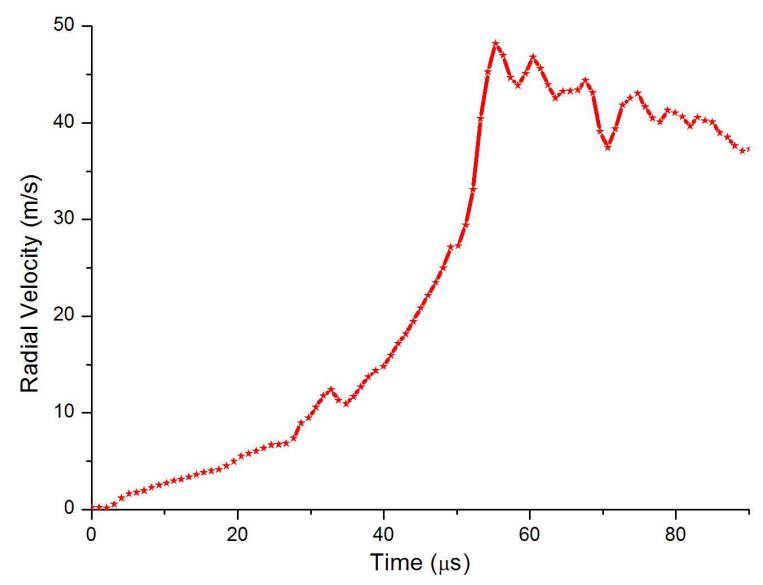

Fig. 6. Typical radial velocity history curves of PMMA ring.

\section{Experimental results and discussion}

\subsection{Experimental observations on fragment form}

\subsubsection{0 aluminum}

The tensile deformation and fracture form of a typical fragmentation are shown in Fig.7. It is found that all the necking and fracture occur under circumferential tension. All the fracture modes of the fragments are the same type which is the typical mode of the free expansion and ductile fragmentation. It confirms that the impact loading experimental technology is feasible that the circumferential tensile stress controls the deformation fragmentation process. Experimental observations on form of all fragments determine that all the fracture points occurred under circumferential tension.

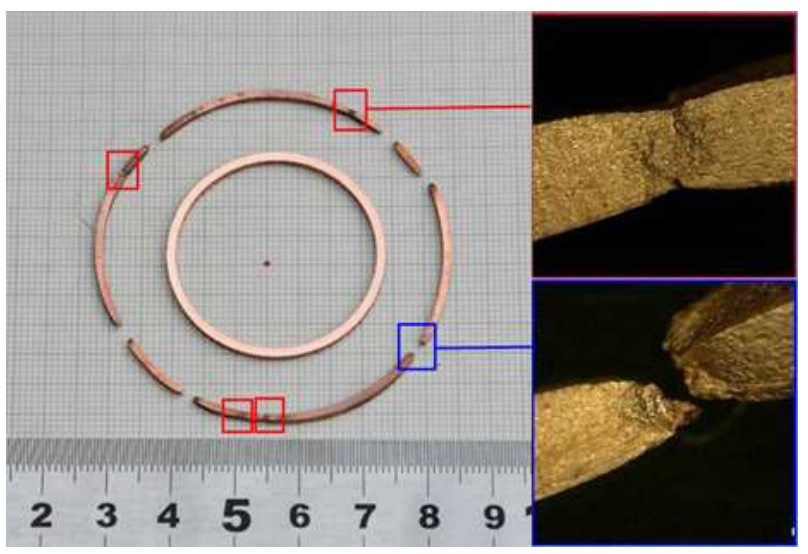

Fig. 7. Recycling fragments, neck and fracture of 1060 aluminum ring.

\subsubsection{PMMA}

The tensile fracture of PMMA material is closer to the brittle fracture, and there is no obvious necking 
phenomenon in the fracture, as shown in Fig.8. Typical PMMA tensile fracture morphology can be roughly divided into three characteristic regions: mirror area (crack source area), fog zone (low-speed expansion zone) and rib-zone (high speed expansion zone).

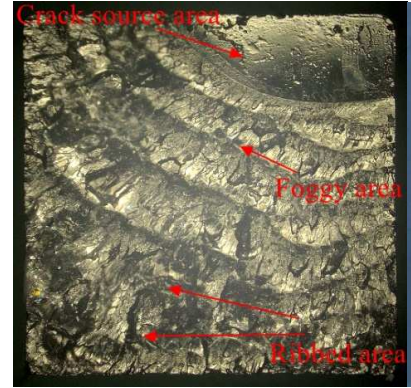

(a)

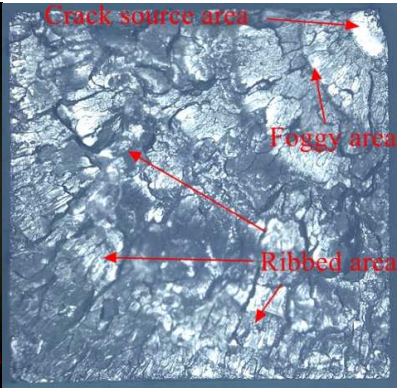

(b)
Fig. 8. (a)The appearance of a low loading rate expanding ring fracture surface; (b) The appearance of a high loading rate expanding ring fracture surface.

In the process of the expanding of the PMMA ring, some fragments contained some non-penetrating cracks shown in Fig. 9. A large number of micro-cracks in the PMMA ring appeared in the expanding process. However, a few cracks could grow and have finally developed into fracture.

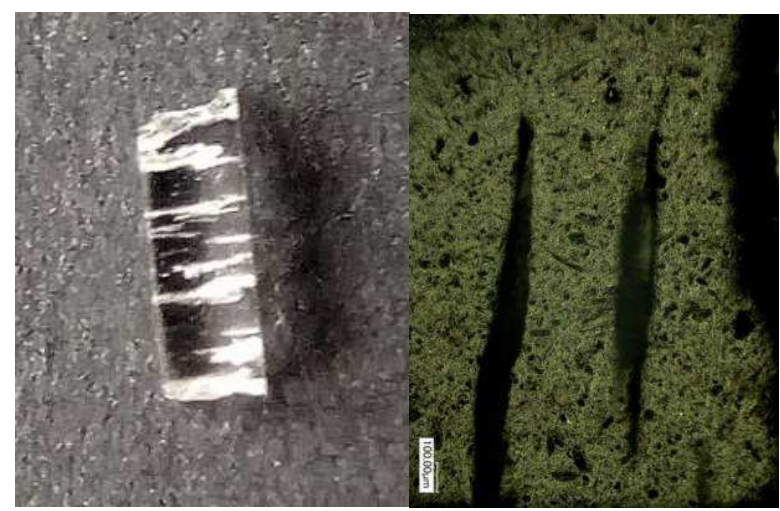

Fig. 9. A typical fragment with arrested internal cracks.

\subsection{Discussion}

From the observations of the fracture morphology and the necking or residual internal cracks of the recovered fragments, it is concluded that the fracture of the rings is caused by the circumferential tensile stress.

The explosive loading and electromagnetic loading, the two different approaches of driving the expanding ring, have a very short boost phase. However, the liquiddriving loading has a relatively long boost phase. The finite element method of fluid-structure interaction was used to simulate the movement, deformation and fracture of the metal ring under the action of high-pressure liquid during the experiment. The finite element model is shown in Fig. 10.

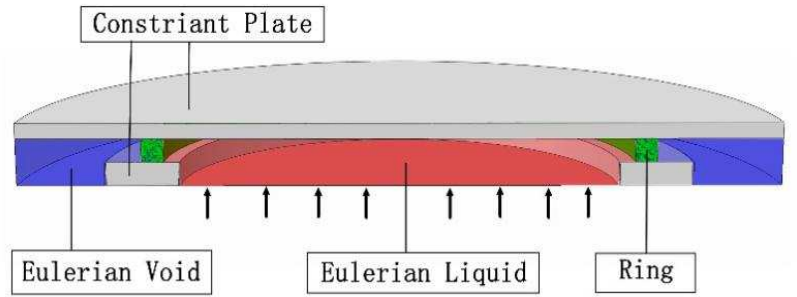

Fig. 10. Finite element model of liquid-driving expanding ring.

The linear elastic model was used to describe the elastic deformation of material. The conventional JohnsonCook thermo-viscoplastic constitutive model was used to describe the dynamic deformation behavior of the material. The Johnson-Cook failure model incorporating a cohesive fracture instability criterion was used to describe the fracture and the separation progress of the material. The type Us-Up model is used for the water. The density of the water is $1000 \mathrm{~kg} / \mathrm{m}^{3}$. The viscosity coefficient of the water is $0.001 \mathrm{~Pa} \cdot \mathrm{s}$. The expanding process is shown in Fig. 11.

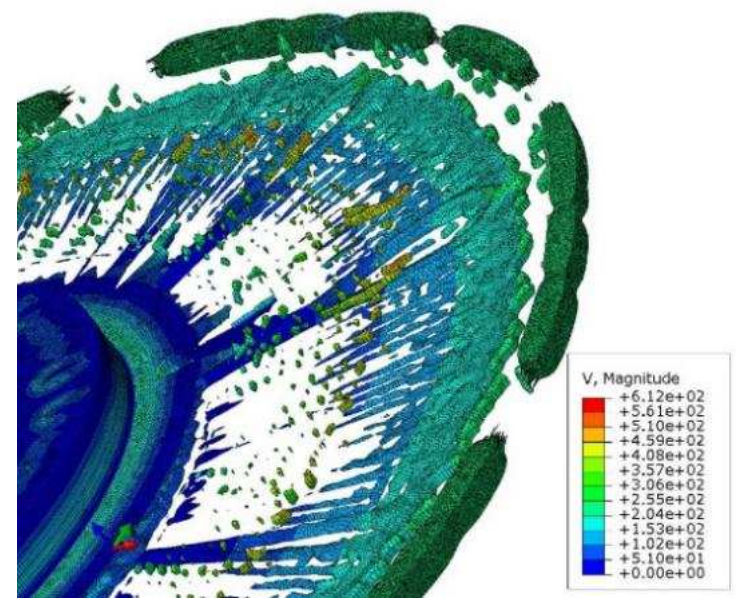

Fig. 11. The typical fracture sense of ring under water driving.

The strain rates of the rings were almost same at the fracture time under liquid-driving loading and the free expansion with the high initial velocity shown in Fig. 12.

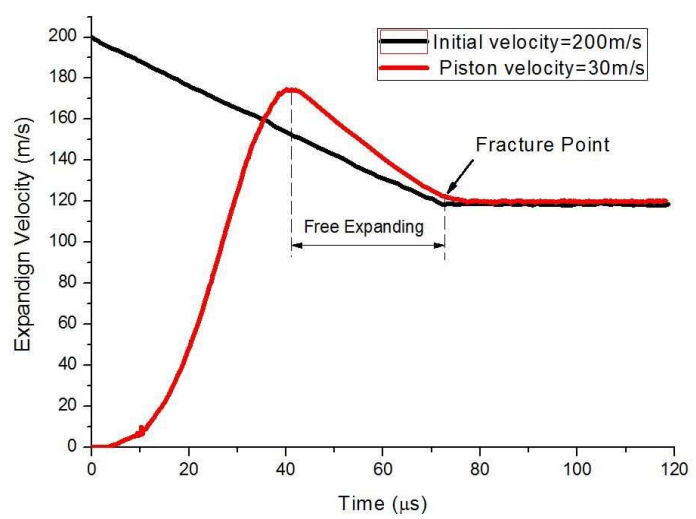

Fig. 12. Radial expanding velocity of specimen. 
Fig. 13 and Fig. 14 shows the fragment process and fragment number of the rings under liquid-driving loading and the free expansion with the high initial velocity are similar.

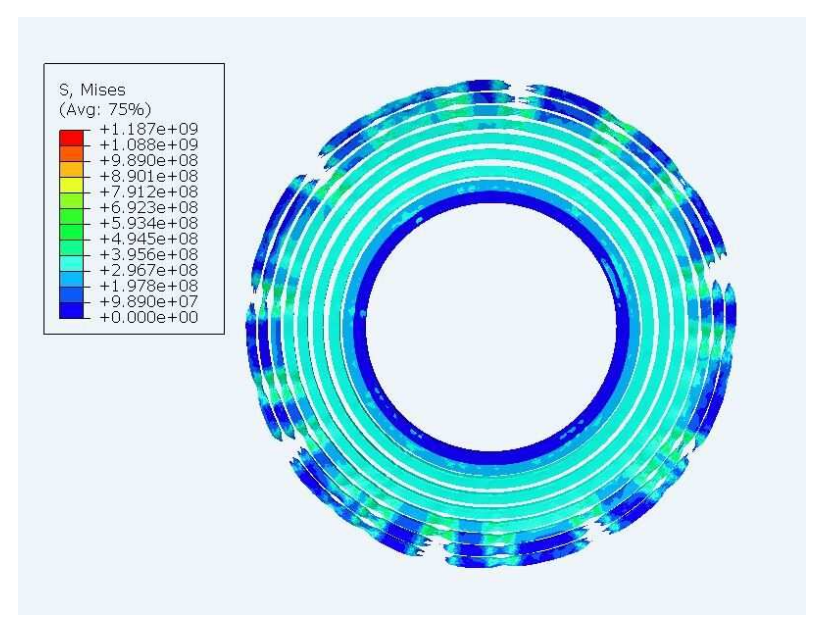

Fig. 13. The fragment process of the ring under liquiddriving loading.

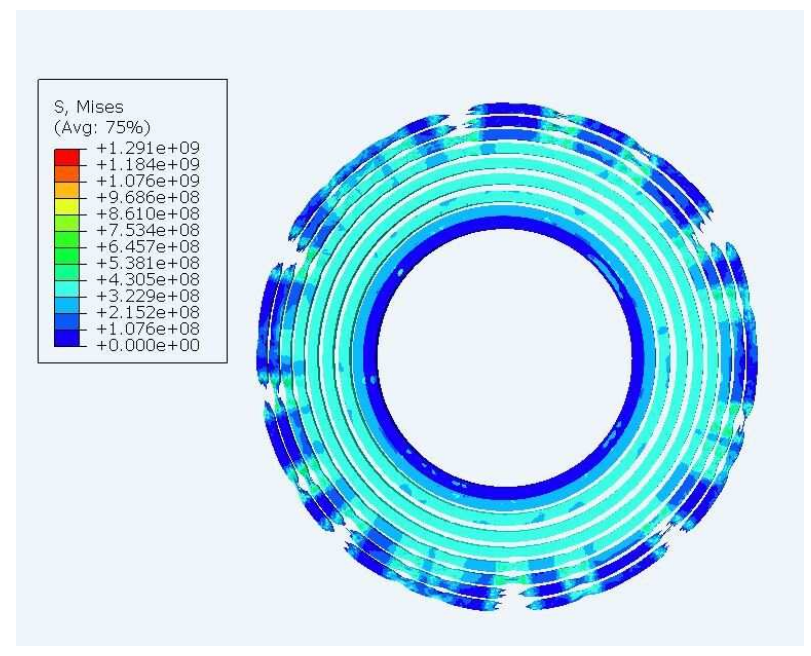

Fig. 14. The fragment process of the ring by the free expansion with the high initial velocity is similar.

\section{Conclusions}

A new loading experimental technology was developed for conducting expanding ring tests, basing on the Split Hopkinson Pressure Bar (SHPB). The tests are useful for the studies on the dynamic tensile deformation and the fracture (fragmentation) properties of materials. The approximately incompressible property of the liquid makes it possible to transfer a low piston-velocity to a very high radial velocity of the specimen, as the sectional areas of the cylinder narrows extremely.

Using the improved liquid-driving expanding ring device, 1060 aluminum rings (ductile materials) and PMMA rings (brittle materials) were fragmented and the fragments were recovered. From the observations of the fracture morphology and the residual internal cracks of the recovered fragments, it is concluded that the fracture of the rings is caused by the circumferential tensile stress.

The research was supported by National Science Foundation of China (11402130, 11390361).

\section{References}

1. Johnson P C, Stein B A, Davh R S. Measurement of dynamic plastic flow properties under uniform stress. in Symposium on the Dynamic Behavior of Materials: ASTM Special Publication (1963)

2. Hoggatt C R, Recht R F. Stress-strain data obtained at high rates using en expanding ring. Experimental Mechanics, 9(10):441-448 (1969)

3. Niordson F I. A unit for testing materials at high strain rates Experimental Mechanics, 5(1):29-32 (1965)

4. Grady D E, Benson D A. Fragmentation of metal rings by electromagnetic loading. Experimental Mechanics, 23(4):393-400 (1983)

5. Gourdin W H. Analysis and assessment of electromagnetic ring expansion as a high-strain-rate test. Journal Applied Physics, 65:411-422 (1989)

6. Zhang H, Ravi-Chandar K. On the dynamics of necking and fragmentation - I. Real-time and postmortem observations in Al 6061-O. International Journal of Fracture, 142(3-4):183-217 (2006)

7. Zhang H, Liechti K M, Ravi-Chandar K. On the dynamics of localization and fragmentation-III. Effect of cladding with a polymer. International Journal of Fracture, 155(2):101-118 (2006)

8. Zhen Yu-xuan, Zhou Feng-hua., Hu Shi-sheng. An SHPB-based experimental technique for dynamic fragmentations of expanding rings. Explosion and Shock Waves, 34(4):483-488 (2014)

9. Zhang Jia, Zheng Yu-xuan, Zhou Feng-hua. Experimental technique for fragmentation of liquiddriven expanding ring. Journal of Ningbo University (Natural Science and Engineering Edition), 30(2):35-38 (2017) 
